\title{
LECTURES ON THE THEORY OF INTEGRATION
}


This page is intentionally left blank

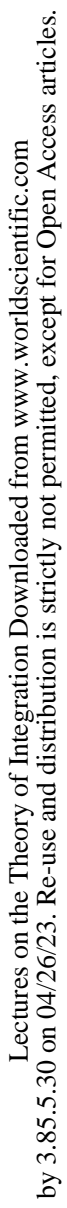


Series in Real Analysis Volume I

\title{
LECTURES ON \\ THE THEORY OF \\ INTEGRATION
}

\author{
Ralph Henstock \\ University of Ulster (Coleraine)
}


Published by

World Scientific Publishing Co. Pte. Ltd.

P.O. Box 128, Farrer Road, Singapore 9128

U. S. A. office: World Scientific Publishing Co., Inc.

687 Hartwell Street, Teaneck NJ 07666, USA

\section{LECTURES ON THE THEORY OF INTEGRATION}

Copyright $\odot 1988$ by World Scientific Publishing Co Pte Ltd.

All rights reserved. This book, or parts thereof, may not be reproduced in any form or by any means, electronic or mechanical, including photo. copying, recording or any information storage and retrieval system now known or to be invented, without written permission from the Publisher.

ISBN 9971-50-450-2

9971-50-451-0 (pbk)

Printed in Singapore by General Printing Services Pte. Ltd. 


\section{CONTENTS}

Preface

0 Prerequisites

CHAPTER 1 INTRODUCTION 1

1 The Riemann and Riemann-Darboux integrals 1

2 Modifications using the mesh and refinement of partitions 4

3 The calculus indefinite integral and the Riemann-complete or generalized Riemann integral

CHAPTER 2 SIMPLE PROPERTIES OF THE GENERALIZED RIEMANN INTEGRAL IN FINITE DIMENSIONAL EUCLIDEAN SPACE 41

4 Integration over a fixed elementary set 41

5 Integration and variation over more than one elementary set 51

6 The integrability of functions of brick-point functions 75

7 The variation set 83

CHAPTER 3 LIMIT THEOREMS FOR SEQUENCES OF FUNCTIONS 90

8 Monotone convergence $\quad 90$

9 Bounded Riemann sums and the majorized (dominated) convergence test $\quad 95$

10 Controlled convergence 102

11 Necessary and sufficient conditions 105

12 Mean convergence and $L^{p}$ spaces 108

CHAPTER 4 LIMIT THEOREMS FOR MORE GENERAL CONVERGENCE, WITH CONTINUITY 123

13 Basic theorems 123

14 Fatou's lemma and the avoidance of nonmeasurable functions 129

CHAPTER 5 DIFFERENTIATION, MEASURABILITY, AND INNER VARIATION 133

15 Differentiation of integrals 133

16 Limits of step functions 149

CHAPTER 6 CARTESIAN PRODUCTS AND THE FUBINI AND TONELLI THEOREMS 162

17 Fubini-type theorems 162

18 Tonelli-type theorems and the necessary and sufficient 
CHAPTER 7 APPLICATIONS

176

19 Ordinary differential equations

176

20 Statistics and probability theory

CHAPTER 8 HISTORY AND FURTHER DISCUSSION

21 Other integrals 191

22 Notes on the previous sections 196

$\begin{array}{ll}\text { REFERENCES } & 199\end{array}$

$\begin{array}{ll}\text { INDEX } & 204\end{array}$ 


\section{PREFACE}

People turn to integration for a variety of reasons and needs, and these can be roughly classified into four kinds. First there are appropriate integral formulae for the evaluation of volumes of solid figures, areas on flat and curved surfaces, and the lengths of certain curves, together with measures of aggregate attributes such as mass, moments of inertia, charge given the charge density, total magnetism given a magnetic density, and so on. Secondly, when solving differential and integral equations in the sciences and elsewhere, the evaluation of integrals is usually vital. Next, continuous linear functionals and self-adjoint (hypermaximal) linear operators over spaces of functions are represented in spectral theory and elsewhere by Stieltjes-type integrals. Finally, integrals are used for distributions in statistics, and these are in turn often used to approximate certain sums. For example, binomial sums are approximated by using suitable normal (or Gaussian) irtegrals.

Students usually begin with the calculus inverse of the derivative, the indefinite integral of Newton, and then turn to the definite integral of Leibnitz, Cauchy, Riemann and Darboux, to name the principal mathematicians involved. These simple integrals satisfy many; much is done in physics and applied mathematics by using the calculus alone, without recourse to more modern ideas. However, it is becoming increasingly clear to scientists in general, especially those interested in statistics, statistical physics, or quantum theory, that such a limitation of the pure mathematical tools is a restriction, and that the use of a more powerful integration process will result in a greater ease of application.

Lesbesgue integration began in a famous paper of 1902, has several useful and interesting properties, and is more powerful in some directions than calculus integration. But a student of its theory endures a complete change of ideas. One approach needs a theory of measure to construct the integral. In another approach the integral is defined directly as a continuous linear functional. But, whatever the approach, the theory is formidable and many find it difficult to understand. Yet the evaluation 
of almost all Lebesgue integrals uses calculus methods.

This curious paradoxical situation often irritated the author when researching in Lebesgue and allied integration theory from 1943 to 1958. Further, even in one dimension it is difficult to estimate a basic element of Lebesgue theory, the measureof the union of open neighbourhoods of known lengths of members of an everywhere dense sequence of points, even when the lengths and points have easy formulae. There is much overlapping of neighbourhoods.

A useful tool for mathematicians and scientists needing advanced integration theory would be a method combining the ideas of the calculus indefinite integral and the Riemann definite integral in such a way that Lebesgue properties can be proved easily, so that the student would not have such a radically fresh start as is needed for Lebesgue integration. Before the 1950's it would have seemed to be an impossible dream. But. independently J. Kurzweil (1957) and the author (1955, 1961) arrived at the same construction, a simple extension of the construction of the Riemann integral. The resulting integral includes the Lebesgue and DenjoyPerron integrals and is the subject of this book. It is one of a whole family of integrals definable by using the Riemann sums over divisions with a limit process that is stronger than the limit as the mesh tends to 0 . The theory has advanced considerably in the 25 years since writing the first book, which is now out of print. Comparison of that pioneering book with the present book shows how proofs have simplified considerably. Further, conditions have been found that are both necessary and sufficient for a sequence of integrable functions to have an integrable pointwise limit, and for the limit to be taken inside the integral sign. The discrete variable can be replaced by a continuous variable, so that necessary and sufficient conditions are found for differentiation and integration under the integral sign. Thus Sections 11, part of 13, 14, 15 in part, and 18 are new, while in Section 19 the Caratheodory theory of ordinary differential equations is improved.

I wish to express my appreciation to Ms. Terri Moss for the excellent typescript, and to many colleagues and students who have checked the lecture notes and have helped to produce a better theory.

R. Henstock 


\section{Prerequisites}

We begin with the usual notation of set theory. A collection of objects is sometimes called a set and sometimes a family, these two terms being interchangeable. If an object $x$ is a member of a set $x$ we write $x \in X$. Sing $(x)$ is the set whose only member is $x$. If every member of $X$ is also a member of a set $Y$ we write $X \subseteq Y$ and $Y \supseteq X$. If $X$ and $Y$ have the same members, $X \subseteq Y$ and $Y \subseteq X$, we regard $X$ and $Y$ as being the same set and write $X=Y$. Otherwise we write $X \neq Y$ and say that $X, Y$ are distinct. If $X \subseteq Y, X \neq Y$, we write $X \subset Y, Y \supset X$. If $M$ is a family of sets $X$ we write,

$$
\underset{M}{U} X{ }_{M} X
$$

for the union and intersection, respectively, of sets $X$ of 11 . The first is the set of all objects each of which lies in at least one of the sets $X$ of $M$. The second is the set of all objects each of which 1 ies in all sets of $M$. Usually $M$ is omitted from the symbols and there are other slight variations. For instance, if $M$ is the collection of $X_{j}$ for $j=1,2, \ldots$, we write

$$
\bigcup_{j=1}^{\infty} x_{j}, \prod_{j=1}^{\infty} x_{j}
$$

Note that these definitions do not need any idea of a limit. The union and intersection of two sets $X_{1}, X_{2}$ are for simplicity written as $X_{1} \cup X_{2}$, $x_{1} \cap x_{2}$, respectively. Two sets with no common member, so that the intersection is the empty set, $\emptyset$, are called disjoint. If each pair of sets from a family $M$ of sets is disjoint, we say that the sets of $M$ are mutually disjoint. If $X$ is a set of real numbers the complement $X$ of $X$ is the set of all real numbers that do not lie in $X$. Thus $X \cap X$ is empty and $X \cup X$ is the set of all real numbers. More generally, $X \backslash Y$ denotes the set of all objects in $X$ that are not in $Y$. If $\left(X_{j}\right)$ is a sequence of sets then we define

$$
\underset{j \rightarrow \infty}{\lim \sup } x_{j}=\bigcap_{m=1}^{\infty} \bigcup_{j=m}^{\infty} x_{j}, \quad \lim _{j \rightarrow \infty} \inf x_{j}={\underset{m=1}{u}}_{j=m}^{\infty} x_{j},
$$


and if they are the same set we write it as

$$
\lim _{j \rightarrow \infty} x_{j}
$$

There is still no idea of a limit in these definitions, even though lim occurs in the names. If $x_{1} \subseteq x_{2} \subseteq \ldots \subseteq x_{j} \subseteq \ldots$ then $\lim _{j} x_{j}=u_{j=1}^{\infty} x_{j}$. We say that $\left(x_{j}\right)$ is monotone increasing. On the other hand, if $x_{1} \supseteq x_{2} \supseteq \ldots \geq x_{j} \supseteq \ldots$ then $\lim _{j} x_{j}=\prod_{j=1}^{\infty} x_{j}$. We say that $\left(x_{j}\right)$ is monotone decreasing. A relation $T$ between the objects $x$ of a set $X$ and the objects $y$ of a set $Y$, is called a one-one correspondence if $T$ satisfies the following requirements.

(0.1) If $x \in X$ there is a $y \in Y$ such that $x T y$;

(0.2) if there is a $z \in Y$ such that $x T z$, then $z=y$;

(0.3) if $y \in Y$, there is an $x \in X$ with $X T y$;

(0.4) if there is a $w \in X$ with $w T y$ then $w=x$.

We also say that $X, Y$ are similar. Using this idea we could define the positive integers as cardinal numbers, and then proceed to define the real and complex number systems. Instead we assume that these systems are already given and will only use the idea of similarity to define countability. A set $X$ is countable if it is similar to the set of all positive integers. Then a subset of a countable set is finite or countable. Also a finite or countable union of finite or countable sets is finite or countable, which is proved by Cantor's diagonal process. But the set of all real numbers in $0 \leq x<1$ is not countable. (For each $x$ in the set can be written as a decimal in the scale of 10 , say, $x=0 . x_{1} x_{2} \ldots x_{n} \ldots$, where $x_{n}$ takes an integer value from 0 to 9 . Some real numbers have a dual representation, for example, $0.50000 \ldots=0.4999 \ldots$. To remove this duality we make the convention that no decimal has $x_{n}=9$ for all $n$ greater than some integer. If $\left(x_{j}\right)$ is a sequence of decimals, with $x_{j}=0 . x_{j 1} x_{j 2} x_{j 3} \ldots x_{j n} \ldots$ we can define a decimal $y=0 . y_{1} y_{2} y_{3} \ldots y_{n} \ldots$ by the rule 


$$
y_{n}=x_{n n}+1\left(0 \leqq x_{n m} \leqq 7\right), y_{n}=1\left(x_{n n}=8,9\right),
$$

so that $y_{n} \neq 9$. Then $y$ differs from each $x_{j}$ in at least the $j$ th. decimal place and $0 \leqq y<1$. Thus no sequence can contain all $x$ in $0 \leqq x<1$.)

The rationals (ratios of integers) are countable, for they are a distinct subsequence of the sequence

$$
0,1 / 1,-1 / 1,2 / 1,-2 / 1,1 / 2,-1 / 2,3 / 1,-3 / 1,2 / 2,-2 / 2,1 / 3,-1 / 3,4 / 1, \ldots
$$

Or put $j / k$ in sequence by writing $j$ and $k$ as integers in the scale of 10 , separated by the /, regard / as the integer 10 in the scale of 11 , interpret $j$ and $k$ now as integers in the scale of 11 , and we have an integer in the scale of 11 . Such an integer cannot be given by any other rational. Thus countability follows, and then the uncountability of the irrationals (those real numbers that are not rational).

In a small part of the book we need a little elementary topology. An open sphere in $n$-dimensional Euclidean space $R^{n}$ with centre $x=\left(x_{1}, \ldots, x_{n}\right)$ and radius $r>0$, is the set of all $y=\left(y_{1}, \ldots, y_{n}\right)$ satisfying

$$
\sum_{j=1}^{n}\left(y_{j}-x_{j}\right)^{2}<r^{2} .
$$

A set $G$ in $R^{n}$ is open if each point of $G$ is the centre of a sphere wholly contained in $G$. A union and a finite intersection of open sets are open. A point $x$ is the 1 imit point of a set $X$ in $R^{n}$ if every sphere, centre $x$, contains a point of $x$ other than $x$. An isolated point of $x$ is a point of $X$ that is not a limit point. The set $X^{\prime}$ of all limit points of $X$ is called the derived set of $X$, and $X \cup X^{\prime}$ is $X^{C}$, the closure of $X$. $A$ set $F$ that contains all its limit points, is called closed. The letter $F$ is taken from ferme, French for 'closed'. A set $X$ is dense-in-itself if every point of $X$ is a limit point of $X\left(X \subseteq X^{\prime}\right)$. A set $P$ that is closed and dense-in-itself $\left(P=P^{\prime}\right)$ is called perfect, and hence the $P$. The complement of an open set is closed, the complement of a closed set is open. An intersection and a finite union of closed sets are closed. 
$A$ set $M$ is dense in a set $X$ if $M \subseteq X \subseteq M^{\prime}$. On the real 1 ine all points $x$ of $X$ for which, for some $\varepsilon>0$, there is an interval $(x-\varepsilon, x)$ or an interval $(x, x+\varepsilon)$, or both, free from points of $X$ (i.e. the set $Y$ of points of $X$ isolated on one or both sides) is a finite or countable set. 